\title{
Clearing the cervical spine of adult victims of trauma
}

\author{
Michael J Clancy
}

The focus of this paper is on the clearance of the cervical spine in those patients who are seriously injured or have the potential to be seriously injured. Specifically excluded from this paper are children. "Whiplash" injury is covered at the end of this paper.

Injuries to the cervical spine occur in $2 \%$ to $12 \%$ of blunt trauma victims, ${ }^{1-4} 10 \%$ to $20 \%$ of patients with serious head injury, ${ }^{56}$ and one in 300 serious motor vehicle accidents. ${ }^{7}$ The emergency physician has a key role in the management of patients with or the potential for cervical spine and cord injuries. First, a spinal injury must be assumed to be present and the spine and cord protected from further injury by immobilisation of the whole spine. Spine and spinal cord injury must be detected and those patients with the potential for further injury from ligamentous damage identified. Cervical spine injury must be excluded in those who are alert and stable. In those who are obtunded immobilisation must continue and others must include the possibility of spine and cord injury in their subsequent investigation and management plans.

The consequences of missing a cervical spinal injury are further avoidable neurological deficits that may lead to death, quadriplegia, and long term disability. The reported incidence of missed or delayed diagnosis of cervical spine injuries is $4.6 \%$ to $8.25 \%{ }^{18}$ but may be as high as $23 \% .{ }^{9}$ It is estimated that for the whole spine the incidence of a secondary neurological deficit, that is where the initial examination revealed an absence of neurological injury with subsequent development of a deficit, is $10.5 \%$ for those with delayed diagnosis compared with $1.4 \%$ for those in whom the fractures were recognised. ${ }^{9}$ However, for the cervical spine this may be as high as $30 \%{ }^{1}$ The financial cost of these patients is considerable. A lifetime cost of caring for a quadriplegic has been estimated at between $\$ 1 \mathrm{~m}$ and $\$ 5 \mathrm{~m}$. ${ }^{10}$

Concern raised by the risk of missing a cervical spine injury and worsening the patient's condition was heightened by anecdotal reports of occult cervical spine fractures. The influential American College of Surgeons Committee on Trauma through the Advanced Trauma Life Support (ATLS) programme ${ }^{11}$ has taught that patients sustaining an injury above the clavicle or a head injury resulting in an unconscious state should be suspected of having an associated cervical spine injury. Any injury produced by high speed vehicles should arouse suspicion of concomitant spine and spinal cord injury. This was coupled with the teaching that "a vertebral column injury should be presumed and immobilisation of the entire patient should be maintained until screening roentgenograms are obtained and fractures or fracture-dislocations are excluded". Apprehension about cervical spine injuries has led to the application of these policies intended for those who are injured or at high risk to those who have minor trauma or who are at low risk of injury. Thus Eliastam et al in 1980 found that $40 \%$ of cervical spinal films were taken for medicolegal reasons. ${ }^{12}$ Immobilisation of the cervical spine is now widespread and liberally applied and has been associated with protocol driven ordering of cervical spine radiographs. ${ }^{13}$ This has led to the liberal use of radiography with, for example, more than $98 \%$ of radiographs ordered in Canadian centres being negative for fracture or dislocation (I Stiell, personal communication).

Clearance of the cervical spine may be said to occur when the clinician is satisfied after appropriate history, examination, and investigation that the risk of an important injury being present is negligible. There is considerable variation in the use of radiography. Thus there is a twofold difference in the rate of use among some Canadian hospitals (I Stiell, personal communication) and there has, for example, been a twofold increase in emergency department cervical spine radiography between 1990 and 1995 at the Bristol Royal Infirmary (internal audit, 1996). This suggests that there are no clear indications as to who should and should not be have radiography. Personal communication with emergency physicians in four major departments in North America (Sunnybrook Hospital, Toronto, Vancouver General Hospital, Oregon Health Sciences University Portland, and the Shock Trauma Centre, Baltimore) confirms that not only is there considerable variation in the indications for radiography but also with regard to which views should be taken for patients who are conscious and alert (fig 1 ).

What criteria should be met for clearance of the cervical spine? The American College of Surgeons Committee on Trauma states "usually..........when no roentgenographic 


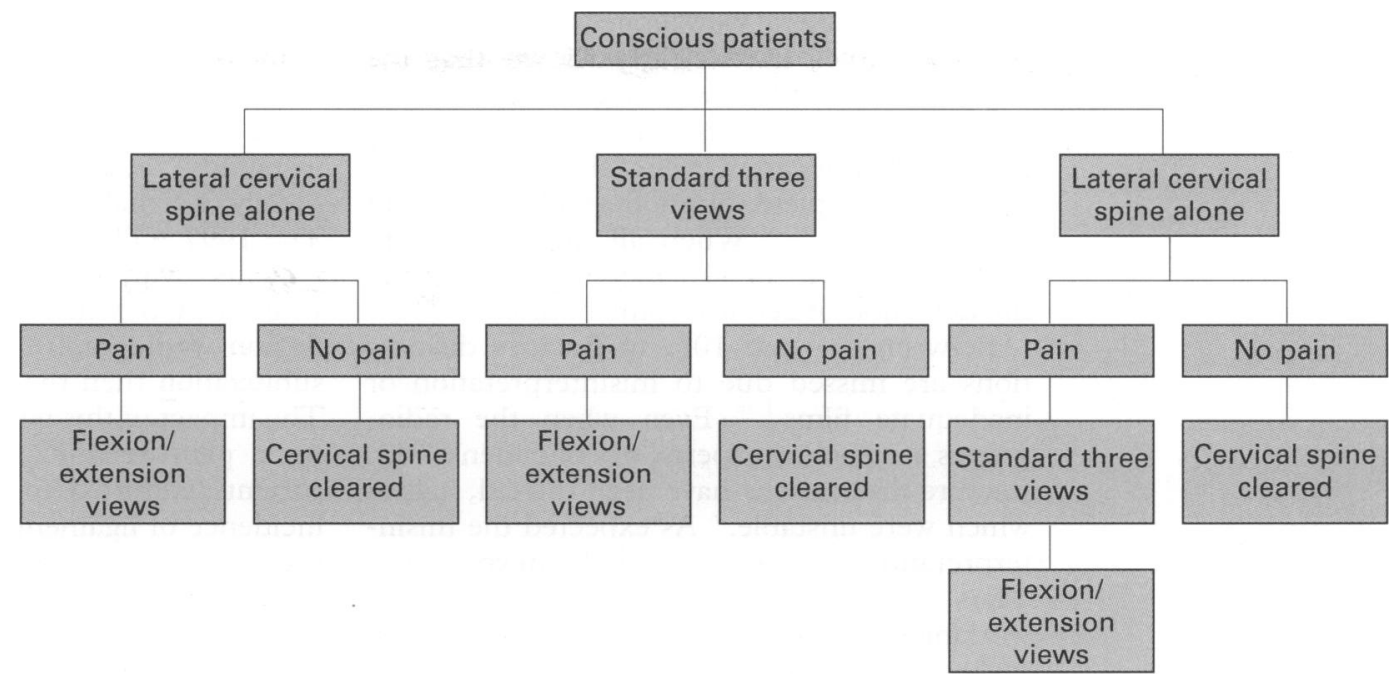

Figure 1 Radiography options for conscious patients.

abnormality has been documented and no symptoms or signs relating to the spine or cord exist"." As Saunders more explicitly states "absent neck pain, obtundation, or neurological findings and normal 3 view $x$-ray films meet an acceptable standard of care". ${ }^{14}$ Yet a telephone survey of 25 intensive care units in the South and West region revealed considerable variation in practice. Nineteen out of 25 would clear the cervical spine of the patient who was still unconscious using only plain radiography and 12 of these 19 would rely on the lateral cervical spine radiography alone. ${ }^{15}$

The more rational use of radiography in alert stable patients would lead to a reduction in unnecessary use, avoidance of prolonged immobilisation, and a substantial saving in the nursing, medical, and radiography time that is currently spent clearing the cervical spine. Potentially there could be shorter emergency department stays and a considerable economic saving.

Patients can be classified into the following: (1) Alert asymptomatic patients with a normal physical examination

There have been many attempts to identify those patients who do not need radiography. ${ }^{1016} 17$ The American College of Radiologists Task Force reviewed the reports of 5719 patients and found no injury in alert asymptomatic patients. ${ }^{18}$ This has tempted some to draw up guidelines. Thus the Royal College of Radiologists state that radiography is not recommended if the patient has no neck pain or tenderness, is fully conscious, not intoxicated, and has no abnormal signs. ${ }^{19}$ These guidelines however pose problems. Where should the tenderness be assessed? In the midline only or also the paraspinal area? What if pain is only demonstrated on movement? What range of movement should be demonstrated? The sixth edition of the ATLS manual (1997) states that with the patient in a supine position the collar can be removed and the spine palpated. ${ }^{20}$ If there is no significant tenderness the patient is asked to voluntarily move his head from side to side. If there is no pain, to voluntarily flex and extend his neck. If this movement is pain free, cervical spine films are not mandatory. Although these guidelines are more specific, neither set address the issue of the masking effect on spinal injuries of other distracting injuries which has been well recognised by a number of authors. ${ }^{16}{ }^{21-24}$ However, a recent study by Velmahos et al of 540 patients failed to find any effect of distracting injuries but concluded that it would require a sample size of between 10000 and 30000 patients to answer the question of whether distracting injuries are important. ${ }^{17}$

Clinicians are looking for more than guidelines in this difficult area over which they are apprehensive, especially if they are inexperienced. They need to be able to predict with confidence who will or will not have a radiological abnormality.

Clinicians are looking for cervical spine decision rules that will demonstrate a sensitivity of 1.0 . It is only by a meticulously designed study recruiting a large number of patients that such rules can be developed as to who does and does not need cervical spine radiography. This is currently being undertaken by Professor Ian Stiell and collaborators in Canada who are recruiting 25000 patients over five years. The study is now entering its third year. Until such rules are available it is likely that there will be widespread variation in the use of radiography in the conscious alert patient and that the threshold for radiography will be a function of individual clinician's experience, what has been taught and level of comfort with not using radiography, which is likely to be low for the junior doctors who see most patients in UK practice.

(2) Patients with cervical spine symptoms or signs All patients with symptoms, that is neck pain or signs relating to the cervical cord or spine, should be immobilised and life threatening pulmonary, cardiovascular, and neurological problems addressed first. It is recommended that the standard three radiography positions (lateral, open mouth, and anteroposterior) be taken and if the C7-T1 junction cannot be demonstrated, swimmer's views, oblique projections, or computed tomography is 
required. ${ }^{20} \mathrm{~A}$ lateral view alone is unacceptable. Several studies have clearly shown that the cross table lateral view in isolation will miss about $15 \%$ of patients with a cervical spine fracture or dislocation even if the films are anatomically complete, of good quality, and read by an expert. ${ }^{25}{ }^{26}$ When all three views are taken a sensitivity of $90 \%$ to $99 \%$ is achieved in the detection of cervical spine injuries. ${ }^{2527}$

Between $4 \%$ and $10 \%$ of fracture dislocations are missed due to misinterpretation or inadequate films. ${ }^{16}$ Even when the radiographs are read by experts, $6 \%$ of patients with fracture dislocations have been missed, half of which were unstable. ${ }^{26}$ As expected the misinterpretation rate is worse with inexperience. Thus Annis et al found that junior doctors working in the emergency department did not make the correct diagnosis in $78 \%$ of the demonstrable fractures of the cervical spine. ${ }^{28}$ This is not helped by the fact that the quality of the lateral cervical spine radiograph may be poor because it is taken with portable equipment and that the top of the first thoracic vertebra may not be visible despite the use of arm traction. ${ }^{17} 2728$ For those patients with neurological deficits, magnetic resonance will provide the best imaging. ${ }^{20}$ In those patients with suspected bony injury, targeted computed tomography is the appropriate investigation. ${ }^{27} 2930$

Those patients who complain of severe neck pain with normal plain radiography are at risk of purely ligamentous injury that could result in instability without any associated fracture. For these patients flexion/extension views are appropriate under the guidance of a knowledgable physician. Contraindications include altered level of consciousness, any subluxation on the lateral film, or any neurological deficit. If C7-T1 is not demonstrated or any area of the cervical spine looks in any way abnormal then computed tomography should be undertaken first. Unsupervised flexion/extension views risk quadriplegia. ${ }^{1}$

How frequent is ligamentous laxity? Lewis et al reported that for a level 1 trauma centre, $13 \%$ of patients having plain radiography of the neck for trauma will have flexion/extension views. ${ }^{31}$ Of these, approximately $8 \%$ (that is $1 \%$ of all patients having cervical spine radiography) will have instability and a third of these will require surgery. It is well recognised that those who subsequently have ligamentous instability requiring surgery may have completely normal initial plain radiography. ${ }^{31-33}$ The 1997 ATLS recommendation is for those patients with neck pain to be asked to voluntarily flex their neck and obtain a lateral flexion radiograph. If this film shows no subluxation then the cervical spine is cleared. The impact of this policy for every patient with neck pain remains to be seen. For those patients admitted to US trauma centres, the incidence of ligamentous laxity seems to be in the order $<0.5 \%$ (S Mirvis, Baltimore and $\mathrm{P}$ Frankel, Portland, personal communication).

\section{(3) Patients with impaired consciousness}

These patients deny the clinician the benefit of complete examination, specifically the neurological examination and the detection of the presence or absence of neck pain. These patients are also at high risk of cervical spine injury. Loss or defect of consciousness is predictive of an unstable cervical spine injury. Altered level of consciousness increased threefold the chance of a spinal fracture ${ }^{34}$ and MacDonald et al found that over half of the patients with cervical spine injury had a Glasgow coma score $<15$ on admission. ${ }^{2}$ Thus it should not be surprising that this group are more likely to suffer missed or delayed detection of cervical spine injury. Indeed, Davis et al found that $70 \%$ of patients with missed or delayed detection of cervical spine injury had altered levels of consciousness. ${ }^{1}$

How are these patients to be cared for? Figure 2 illustrates the alternative practices in the four North American centres previously listed. Patients may be kept immobilised until they regain consciousness. The benefits of this should be continued protection of the cervical spine and cord until the patient is alert at which stage a full examination can take place. However, cervical collars provide incomplete immobilisation, produce full thickness ulcers beneath them in $55 \%$ of patients who have had them applied for greater than five days, ${ }^{35}$ and there is increase in the nursing workload

Unconscious patient

Standard three views +1computed tomography of $\mathrm{Cl}-\mathrm{C} 3$

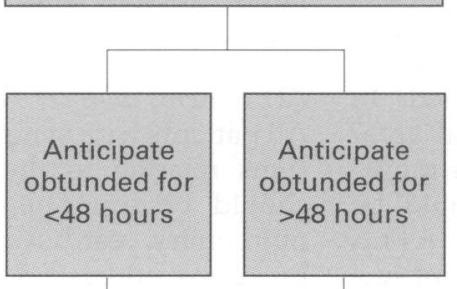

Continue immobilisation

immobilisation

Figure 2 Management options for the unconscious patient.

Continue immobilisation until regains consciousness
Search for

ligamentous laxity (within 48 hours or at 7-10 days)

If normal discontinue immobilisation 
because of repeated log rolling. Patients may be at greater risk of pneumonia and thromboembolism. Continued immobilisation also risks delay in detection and treatment of an unstable spinal injury.

In the light of these disadvantages it would seem sensible to limit immobilisation to those who are or likely to remain unconscious for a short period only (less than 48 hours). For those likely to remain unconscious for longer, early clearance of the cervical spine and discontinuation of cervical spine immobilisation seems desirable. How can this be achieved? Three approaches have been used:

(A) Use of normal three view plain radiography alone. Thus MacDonald et al in a retrospective review of 775 motor vehicle accident victims concluded that complete three view radiography could be used to clear the spine at the risk of missing fractures in fewer than $1 \%$ of patients. ${ }^{2}$ This approach risks failing to detect ligamentous laxity or those fractures not detectable with plain radiography. However, this approach is supported by the American College of Surgeons with the rider of appropriate evaluation of the patient by a neurological or orthopaedic surgeon. ${ }^{20}$

(B) Three view plain radiography plus routine computed tomography of $\mathrm{C} 1-\mathrm{C} 3$. This is based on the fact that occult fractures occur commonly at $\mathrm{C} 1-\mathrm{C} 2$ and that Frye et al found that in those patients with a Glasgow coma score <12, two out of 93 had fractures of C1-C3, of which one required surgery. ${ }^{36}$ Similarly, Kirshenbaum et al found four out of 50 had fractures of C1-C2 not visible on plain radiography. ${ }^{37}$

(C) Plain radiography plus actively searching for ligamentous laxity by stressing the cervical spine. The Baltimore protocol is illustrated in fig 3. This relies on normal view three view plain radiography and uses an erect lateral cervical spine where the weight of the patient's head is used to apply an axial load to the spine. It is well recognised that disruption of the posterior ligamentous complex may give minimal radiological signs on a supine film but that an erect lateral film will show the characteristic kyphotic hyperangulation. Alternatively passive flexion/extension views under fluoroscopy have been reported by Davis et al who demonstrated that none of the 116 patients came to any harm. ${ }^{35}$ However, given the small number of patients studied and the known frequency of cervical spine injury, this study should not be reassuring with regards to the safety of the procedure. Flexion/extension views may be considered potentially dangerous because plain radiography may offer no clue as to the presence of an extradural haematoma or prolapsed disc. There is always the risk of an unexpected slip of the unstable spine during the procedure. The timing of the search for ligamentous laxity varies. Thus the Baltimore protocol is undertaken within 48 hours. Others undertake flexion/extension views seven to 10 days after injury if the patient remains obtunded.
What measures can be used to improve the detection of cervical spine injuries? The widespread practice of cervical spine immobilisation should not be changed. Although large numbers turn out to be unnecessarily immobilised it serves initially to remind all carers to consider the spine. Butman et al concluded that the current practice of prehospital immobilisation based on the mechanism of injury or the presence of other injuries has contributed to the decline in the frequency of spinal cord injury and the practice should not be changed. ${ }^{38}$

All doctors who request cervical spine radiography should be able to interpret the films competently. For the high risk unconscious group of patients these films should be read by a radiologist with an interest in this field. All films must be of good quality and the cervical spine must be demonstrated in its entirety. Given that most patients who are unconscious will have computed tomography of their head, one could sensibly argue for routine scanning of $\mathrm{C} 1$ through to $\mathrm{C} 3$. Anecdotally this practice is now happening within the UK.

How hard should one look for ligamentous laxity? The survey of the intensive care units within the South and West region revealed that no department routinely searched for ligamentous laxity in the unconscious patient. There is no easy answer to this question. How thoroughly one looks for this type of injury is likely to depend on its frequency in the population and local expertise and interest in this problem. Apart from the options already mentioned, one solution might be routine magnetic resonance imaging for those patients who remain unconscious after 48 hours. Benzel et al scanned 174 post-traumatic patients who did not appear to harbour disruption of spinal integrity on the basis of routine radiography. ${ }^{39}$ None had a clinically obvious injury; 62 (36\%) had soft tissue abnormalities identified by magnetic resonance imaging. Of these 62 , one underwent surgery, 35 were placed in a cervical collar for one month, and 27 placed in a Minerva jacket for at least two months. The spines of patients whose magnetic resonance images were negative were considered clear and no patient suffered complications of a spinal or neurological nature. Clearly this imaging modality needs further evaluation but has great potential in this difficult area.

\section{Whiplash injury}

The evidence relating to the diagnosis and management of whiplash injuries was reviewed by a specially commissioned taskforce in Quebec which reported in $1995 .{ }^{40}$ They defined whiplash as "an acceleration-deceleration mechanism of energy transfer to the neck. It may result from rear-end or side impact motor vehicle collisions but can also occur during diving or other mishaps. The impact may result in bony or soft tissue (whiplash injury), which in turn may lead to a variety of clinical manifestations (whiplash associated disorders, WAD)". They usefully classified WAD into four levels (table 1) and made recommendations regarding imaging and treatment (fig 4). 
Thus the patient with grade I WAD who is fully alert but complains of neck pain, stiffness, or tenderness with a normal range of motion and absence of point tenderness, does not usually require radiography. Grades II and III WAD need "baseline" radiography and further imaging is not considered until these patients are reviewed as outpatients, unless initial plain radiography is equivocal. Grade IV WAD mandates immediate referral and likely further imaging. Clearly these recommendations are different from those made earlier in this paper and reflect the more benign nature of whiplash injuries. It should be noted, however, that these are only recommendations (made by an expert group in the absence of firm evidence), which like many of those elsewhere in this paper remain untested.

\section{Conclusion}

The clearance of the cervical spine continues to tax emergency physicians, nevertheless they are becoming more discriminatory in the their use of plain radiography. Cervical spine radiography rules for stable and alert trauma patients developed along the same lines as the Ottawa ankle rules ${ }^{41}$ are eagerly awaited and are likely to have a major impact on the way the

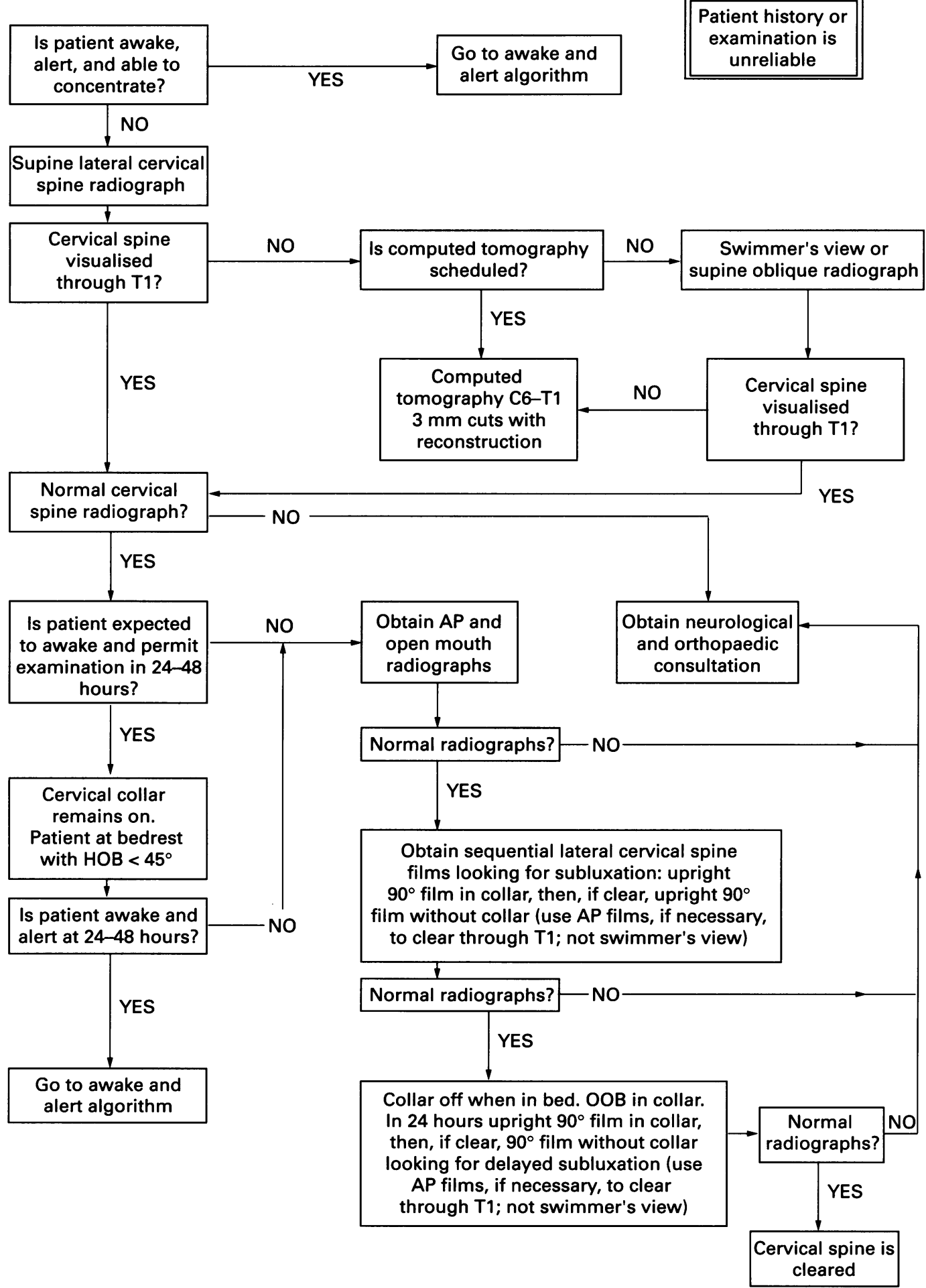

Figure 3 Guidelines for clearing the cervical spine; $A P=$ anteroposterior; $H O B=$ head of bed; $O O B=$ out of bed. 
Table 1 Proposed clinical classification of whiplash associated disorders (Reproduced with permission of Lippincott Williams and Wilkins from WO Spitzer et al. Scientific monograph of the Quebec Task Force on whiplash associated disorders. Spine 1995;20(suppl):8S)

\begin{tabular}{|c|c|}
\hline Grade & Clinical presentation \\
\hline 0 & $\begin{array}{l}\text { No complaint about the neck } \\
\text { No physical sign(s) }\end{array}$ \\
\hline-- & ---------------------- \\
\hline I & $\begin{array}{l}\text { Neck complaint of pain, stiffness, or tenderness only } \\
\text { No physical sign(s) }\end{array}$ \\
\hline II & Neck complaint AND musculoskeletal sign(s) ${ }^{\star}$ \\
\hline III & Neck complaint AND neurological sign(s) $\dagger$ \\
\hline$\overline{I V}--$ & Neck complaint AND fracture or dislocation \\
\hline
\end{tabular}

${ }^{\star}$ Musculoskeletal signs included decreased range of motion and point tenderness.

†Neurological signs include decreased or absent deep tendon reflexes, weakness, and sensory deficits.

Symptoms and disorders that can be manifest in all grades include deafness, dizziness, tinnitus, headache, memory loss, dysphagia, and temporomandibular joint pain.

Dotted lines indicate limits of terms of reference of Task Force.

cervical spine is cleared. Magnetic resonance imaging offers a way forward for the obtunded patient but more studies are needed. In the mean time, guidelines developed from the best available (but inadequate) evidence and clinical judgment will have to suffice.

The author wishes to thank the Faculty of Emergency Medicine for the award of the Alison Gourdie prize and in particular Barry McClellan of Toronto, Doug McKnight of Vancouver, Jerris Hedges of Portland, and Stuart Mirvis of Baltimore.

\section{Questions relating to this article}

(1) List the contraindications to flexion/ extension views of the cervical spine.

(2) Do you know how to evaluate guidelines? If not refer to "Papers that tell you what to do (guidelines)" in How to Read a Paper. The Basics of Evidence Based Medicine by Trisha Greenhalgh. London: BMJ Publishing Group, 1997. (3) Read the scientific monograph of the Quebec Task Force on whiplash associated disorders: redefining whiplash and its management by Spitzer et al. ${ }^{40}$ Are thes guidelines useful? Do you need to change your current practice? Conflict of interest: none.

Funding: none.

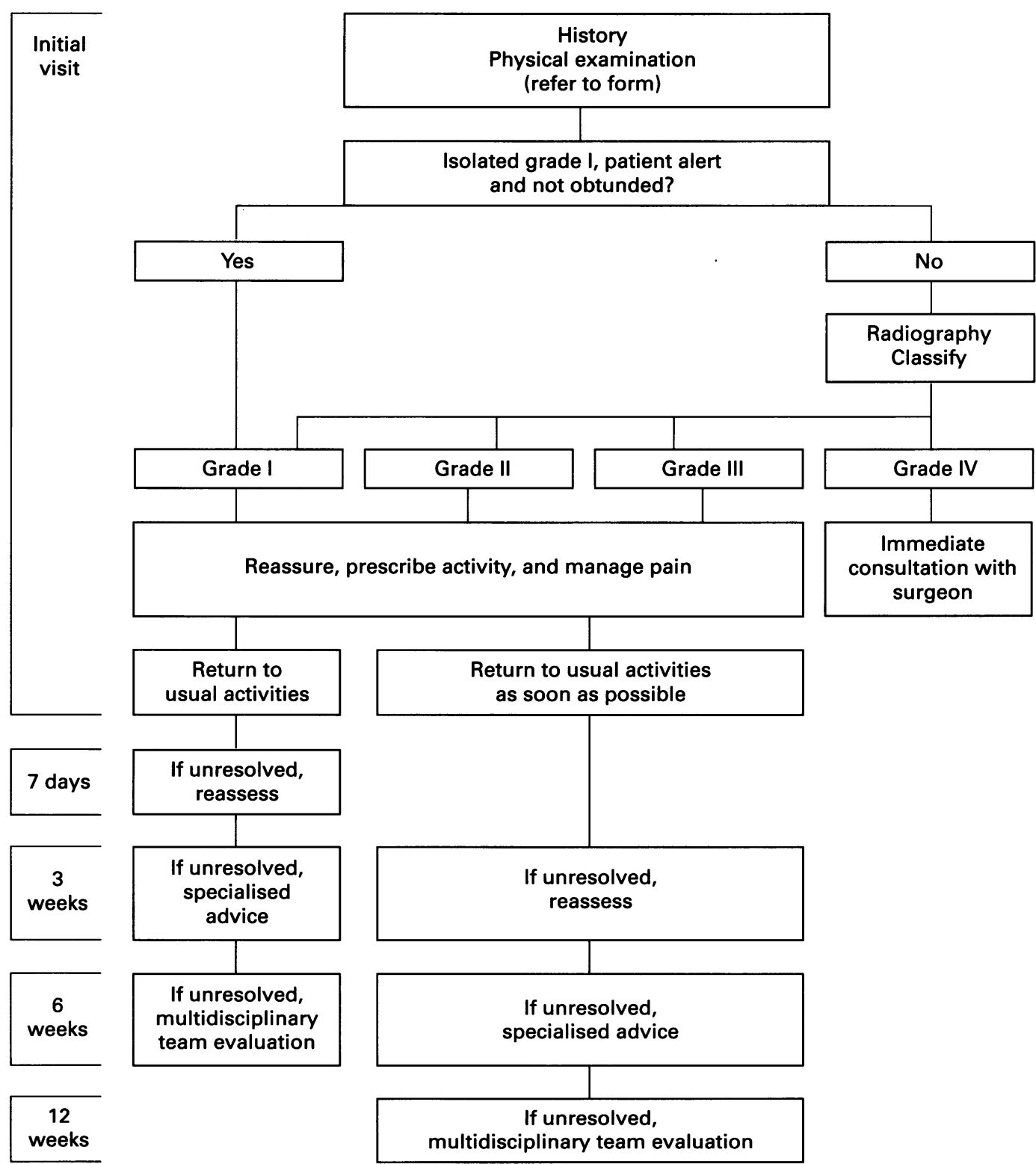

Figure 4 Quebec guidelines for patient care. (Reproduced with permission of Lippincott Williams and Wilkins from WO Spitzer et al. Scientific monograph of the Quebec Task Force on whiplash associated disorders. Spine 1995;20(suppl):8S.) 
1 Davis JW, Phreaner DL, Hoyt DB, et al. The etiology of missed cervical spine injuries. $\mathcal{F}$ Trauma 1993;34:342-6.

2 MacDonald RL, Schwartz ML, Mirich D, et al. Diagnosis of cervical spine injury in motor vehicle crash victims: how many $x$-rays are enough? $\mathcal{F}$ Trauma 1990;30:392-7.

3 Ross SE, O'Malley KF, de Long WG, et al. Clinical predictors of unstable cervical spine injury in the multiply injured patients. Injury 1992;23:317-9.

4 Cohn SM, Lyle WG, Linden CH, et al. Exclusion of cervical spine injury: a prospective study. 7 Trauma 1991;31:570-4.

5 Roberts JR. Trauma of the cervical spine. Topics in Emergency Medicine 1979;1:63.

6 Rockswold GL. Evaluation and resuscitation in head trauma. Minn Med 1981;64:81.

7 Heulke DF, O'Day J, Mendleson RA. Cervical injuries suffered in automobile crashes. $¥$ Neurosurg $1981 ; 54: 316-22$

8 Gerrelts BD, Petersen EU, Mabry J, et al. Delayed diagnosis of cervical spine injuries. $\mathcal{F}$ Trauma 1991;31:1622-6.

9 Reid DC, Henderson R, Saboe L, et al. Etiology and clinical Reid DC, Henderson R, Saboe $L$, et al. Etiology and clinical

10 Bachulis BL, Long WB, Hynes GD, et al. Clinical indications for cervical spine radiographs in the traumatized patient. Am f Surg 1987;153:473-7.

11 American College of Surgeons. Committee on Trauma. Advanced life support course for physicians. Chicago: American College of Surgeons, 1993.

12 Eliastam M, Rose E, Jones $\mathrm{H}$, et al. Utilization of diagnostic radiologic examinations in the emergency department of teaching hospital. $\mathcal{F}$ Trauma 1980;20:61-6.

13 Mirvis SE, Diaconis JN, Chirco PA, et al. Protocol driven radiologic evaluation of suspected cervical spine injury: efficacy study. Radiology 1989;170:831-4.

14 Saunders RL. The standard of care-cervical spine trauma [editorial comment]. $\mathcal{F}$ Trauma 1993;34:346.

15 Gupta KJ, Clancy M. Discontinuation of cervical spine immobilisation in unconscious patients with trauma in immobilisation in unconscious patients with trauma in intensive care units. Telephone survey of practic
South and West region. $B M \mathcal{F} 1997 ; 314: 1652-5$.

16 Ringenberg BJ, Fisher AK, Urdaneta LF, et al. Rational Ringenberg BJ, Fisher AK, Urdaneta LF, et al. Rational
ordering of cervical spine radiographs following trauma. Ann Emerg Med 1988;17:792.

17 Velmahos GC, Theodorou D, Tatevossian R, et al. Radiographic cervical spine evaluation in the alert asymptomatic blunt trauma victim: much ado about nothing? $f$ Trauma 1996;40:768-74.

$18 \mathrm{Kathol}$ MH. Cervical spine trauma. What is new? Radio Clin North Am 1997;35:507-32.

19 Royal College of Radiologists. Guidelines for the use of the radiology department. London: Royal College of Radiologists, 1995

20 American College of Surgeons. Committee on Trauma. Advanced life support course for physicians. 6th Ed. Chicago: American College of Surgeons, 1997.

21 Hoffman JR, Schriger DL, Mower W, et al. Low risk criteria for cervical radiography in blunt trauma: a prospective study. Ann Emerg Med 1992;21:1454-60.

22 Roth BJ, Martin RR, Foley K, et al. Roentgenographic evaluation of the cervical spine. A selective approach. Arch Surg 1994;129:643.

23 McNamara RM, Heine E, Esposito B. Cervical spine injury and radiography in alert high risk patients. $\mathcal{f}$ Emerg Med 1990;81:77.
24 Spain DA, Trooskin DZ, Flanebaum L, et al. The adequacy and cost effectiveness of routine resuscitation cervical spine radiographs. Ann Emerg Med 1990;19:272.

25 Streitwieser DR, Knopp R, Wales LR, et al. Accuracy of standard radiographic views in detecting cervical spine fractures. Ann Emerg Med 1993;12:538-42.

26 Woodring JH, Lee C. Limitations of cervical radiography in the evaluation of acute cervical trauma. $\mathcal{F}$ Trauma 1993;34: 32-9.

27 Ross SE, Schwab CW, Eriberto TD, et al. Clearing the cervical spine: initial radiologic evaluation. F Trauma 1987;27: 1055-9.

28 Annis JA, Finlay DB, Allen MJ, et al. A review of cervical spine radiographs in casualty patients. $\mathrm{Br} \mathcal{F}$ Radiol $1987 ; 60$ : 1059-61

29 Borock EC, Grabram SG, Jacobs LM, et al. A prospective analysis of a two year experience using computerised tomography as an adjunct for cervical spine clearance. $\mathcal{f}$ Trauma 1991;31:1001-6.

30 Woodring $\mathrm{JH}$, Lee $\mathrm{CL}$. The role and limitations of computed tomographic scanning in the evaluation of cervical trauma. F Trauma 1992;33:698-708.

31 Lewis LM, Docherty M, Ruoff BE, et al. Flexion extension views in the evaluation of cervical spine injuries. Ann Emerg Med 1991;20:117-21.

32 Fazl M, LaFebvre J, Willinsky RA, et al. Posttraumatic ligamentous disruption of the cervical spine, an easily overlooked diagnosis: presentation of three cases. Neurosurgery 1990;26:674-8.

33 Gehweiler JA, Osborne RL, Becter RF. The radiology of vertebral trauma. Philadelphia: WB Saunders, 1980: 228-39.

34 Kreipke DL, Gillespie KR, McCarthy MC, et al. Reliability of indications for cervical spine films in trauma patients. $\mathcal{f}$ Trauma 1989;29:1438-9.

35 Davis JW, Parks SN, Detlefs CL, et al. Clearing the cervical spine in obtunded patients: the use of fluoroscopy. $\mathcal{F}$ Trauma 1995;39:435-8.

36 Frye G, Wolfe T, Knopp R, et al. Intracranial hemorrhage as a predictor of occult cervical spine fracture. Ann Emerg Med 1994:23:435-8.

37 Kirshenbaum KJ, Nadimpalli SR, Fantus R, et al. Unsuspected upper cervical spine fractures associated with significant head trauma: role of CT. $\mathcal{f}$ Emerg Med 1990;8:183-98

38 Butman AM, Schelble DT, Vomacka BA. The relevance of the occult cervical spine controversy and mechanism of injury to prehospital protocols:a review of the issues and injury to prehospital protocols:a review of the issues and 33.

39 Benzel EC, Hart BL, Ball PA, et al. Magnetic resonance imaging for the evaluation of patients with occult cervica spine injury. I Neurosurg 1996;85:824-9.

40 Spitzer WO, Skovron ML, Salmi LR, et al. Scientific monograph of the Quebec Task Force on whiplash associated disorders. Spine 1995;20(suppl):8S.

41 Stiell IG, McKnight RD, Greenberg GH, et al. Implementation of the Ottawa ankle rules. $¥ A M A 1994 ; 241: 827-32$. 